\title{
A 14-day follow-up of adult non-malarial fever patients seen by mobile clinics in rural Malawi
}

\author{
Kimberly A. Baltzell ${ }^{1,2}$, Teresa Bleakly Kortz ${ }^{3,1}$, Alden Blair ${ }^{1}$, Ellen Scarr ${ }^{2}$, Andrew M. Mguntha ${ }^{4}$, \\ Gama Bandawe ${ }^{5}$, Ellen Schell ${ }^{2,6}$, Sally H. Rankin ${ }^{2}$
}

1. Institute for Global Health Sciences, University of California, San Francisco, San Francisco, CA, USA

2. Department of Family Health Care Nursing, University of California San Francisco, San Francisco, CA, USA

3. Department of Pediatrics, University of California, San Francisco, San Francisco, CA, USA

4. ENANDY Research Consultancy, Blantyre, Malawi

5. Department of Biological Sciences, Malawi University of Science and Technology, Malawi

6. Global AIDS Interfaith Alliance, San Rafael, CA, USA

\section{Background}

\section{Abstract}

While health providers consistently use malaria rapid diagnostic tests to rule out malaria, they often lack tools to guide treatment for those febrile patients who test negative. Without the tools to provide an alternative diagnosis, providers may prescribe unnecessary antibiotics or miss a more serious condition, potentially contributing to antibiotic resistance and/or poor patient outcomes.

Methods

This study ascertained which diagnoses and treatments might be associated with poor outcomes in adults who test negative for malaria. Adult patients for rapid diagnostic test of malaria seen in mobile health clinics in Mulanje and Phalombe districts were followed for 14 days. Participants were interviewed on sociodemographic characteristics, health-seeking behaviour, diagnosis, treatment and access to care. Mobile clinic medical charts were reviewed. Two weeks ( \pm 2 days) following clinic visit, follow-up interviews were conducted to assess whether symptoms had resolved.

Results

Initially, 115 adult patients were enrolled and $1(0.88 \%)$ was lost to follow-up. Of the 114 adult patients remaining in the study, 55 $(48 \%)$ were seen during the dry season and 59 (52\%) during the wet season. Symptoms resolved in $90(80 \%)$ patients at the 14-day follow-up visit $(n=90)$ with the rest $(n=24)$ reporting no change in symptoms. None of the patients in the study died or were referred for further care. Almost all patients received some type of medication during their clinic visit $(98.2 \%)$. Antibiotics were given to $38.6 \%$ of patients, and virtually all patients received pain or fever relief $(96.5 \%)$. However, no anti-malarials were prescribed.

Conclusions

Mobile clinics provide important health care where access to care is limited. Although rapid tests have guided appropriate treatment, challenges remain when a patient's presenting complaint is less well defined. In rural areas of southern Malawi, simple diagnostics are needed to guide treatment decisions.

Key Words: Non-malarial fever, Malawi, febrile illness, mobile clinics, diagnostic tools, antibiotic resistance

\section{Introduction}

Malaria has long been thought to be the cause of fevers in many areas in sub-Saharan Africa. However, with the introduction of relatively accurate malaria rapid diagnostic tests (mRDT), fewer health providers are treating malaria solely on clinical symptoms ${ }^{1}$. While this has improved outcomes for those who have malaria, those who test negative by mRDT are left without a clear diagnosis or treatment plan². Further, misdiagnosing fevers has important negative personal and public health consequences. The misdiagnosis of fever results in non-treatment of the actual etiology-upper respiratory tract infections (typically viral), lower respiratory tract infections (including bacterial/viral/fungal pneumonias, bronchiolitis and tuberculosis [TB]), gastroenteritis, schistosomiasis, intestinal nematode infections, genitourinary tract infections, meningitis, bacteraemia, and HIV and its associated illnesses-which may lead to significant morbidity and death ${ }^{3}$. Misdiagnosis thus leaves a patient vulnerable to worsening of the underlying illness, and allows for transmission of communicable diseases, such as TB. Consideration of the differential diagnoses of febrile illnesses is critical, though it is often difficult to differentiate the etiology of the fever in the absence of definitive diagnostic tests ${ }^{4}$. Although there is research showing that fevers in hospitalised African adults and children are often associated with HIV-induced immunosuppression, upper respiratory illness and environmental exposures such as leptospirosis, rickettsiosis and brucellosis, little is known about causes of fever in primary health care facilities, where most patients are seen in low income countries ${ }^{5}$. Further, patients with mild respiratory symptoms and low-grade fever are often treated with antibiotics, despite the absence of signs/symptoms of a bacterial infection, and inappropriate use of antibiotics is known to contribute to the development of antibiotic resistance $^{6}$. Such inappropriate treatment may indicate a lack of provider awareness of other causes of fever and how to manage them, a distrust in mRDT validity, or concern for patient well-being given limited access to health care services and follow-up.

Without another alternative diagnosis to offer or the ability to follow-up with a patient, health care providers may fail to prescribe appropriate treatment (or offer inappropriate treatment) for non-malarial fevers. The first step in improving management of febrile illness is to determine patient outcomes for mRDT-negative patients. This study explored clinical outcomes in mRDT-negative febrile adults 
seen by mobile health clinics in southern Malawi. The overall objective of the study was to describe factors associated with incomplete symptom resolution in adult patients who test negative for malaria.

\section{Methods \\ Overview}

This study aimed to identify and describe mRDT-negative adult patients who are at risk for worsening illness in the 2 -week period following a visit to a mobile health clinic. This article contains data from a larger prospective cohort study of people with non-malarial fevers who received care at the Global AIDS Interfaith Alliance (GAIA) mobile clinics in Muloza (Phalombe District) or Nkanda (Mulanje District), shown in Figure 1, during either the dry or wet seasons (August-September 2016 and November-December 2016, respectively). The methods and rationale of the parent study are reported on elsewhere ${ }^{7}$. Briefly, participants who presented at the clinics with a complaint of general illness and an axillary temperature of $37.5^{\circ} \mathrm{C}$ or reported a history of fever in the last 48 hours and were mRDT-negative were eligible for inclusion in the study. Patients were tested using the SDBioline mRDT which reports an overall sensitivity of $99.5 \%$ for Plasmodium falciparum and $92.6 \%$ for non- $P$. falciparum infection, and $98 \%$ specificity for P. falciparum and $100 \%$ for non- $P$. falciparum infections ${ }^{8}$. Patients in extremis or obtunded, already enrolled in the study, known to be unavailable or unreachable for follow-up, and who did not speak English or Chichewa fluently were excluded from recruitment.

Once identified, study staff fluent in Chichewa approached eligible patients to explain the purpose of the study and gain written consent. Patients who agreed to participate were then interviewed on sociodemographic characteristics, health-seeking behaviour, diagnosis, treatment, and access to care. Data were also extracted from GAIA mobile clinic charts. Two weeks ( \pm 2 days) following the interview, local, trained field research staff went to the participants' homes to conduct follow-up interviews and assess the status of the presenting complaint and associated information.

This study reports on findings from all patients over the age of 14 years $(n=115)$ who consented to participate during the course of the research. Assent was obtained for patients between the ages of 14 and 18 years. Presenting complaints were self-reported while clinical diagnoses, treatment and medication regimens were collected via clinical logbooks. HIV status was also self-reported because HIV diagnoses were tracked in separate logs that were unavailable to the researchers at the time of the study. Food insecurity was defined as having a family member or personal experience missing a meal when hungry in the past week. Medication non-compliance was defined as having, for any reason, not completed the entire course prescribed by GAIA, which was given to the patient in full as part of GAIA's service. Participant's medical status at follow-up was self-reported and defined as having resolved or got better compared with no change or worsening of symptoms.

Data analysis was completed using the $\mathrm{R}$ statistical package version 3.2.4 (The R Foundation, available from: https:// www.r-project.org/foundation/). Initially, descriptive statistics were calculated to better understand cohort level demographic breakdowns. Next, bivariate analyses were run to explore the associations between participant characteristics and their symptom status at follow-up (resolved/better vs no change/worsening). The Wilcoxon signed-rank test was used for continuous exposure variables as none were normally distributed. Fisher's exact test was used for categorical exposures due to the small sample size and occurrences of zero and cell counts of $\leq 5$. Measures of association include both P-values and unadjusted odds ratios (UOR) and 95\% confidence intervals (95\% CI) to show both direction and magnitude of associations.

\section{Ethical approval}

This study received ethical approval from the University of California San Francisco (UCSF) Human Research Protection Program (approval \#16-19187) and the Malawi National Health Sciences Research Committee (Protocol \#16/5/1594).

\section{Results}

During the study period, a total of 2893 patients (adults and children) were screened, 489 met inclusion criteria and 401 were enrolled. Of the enrolled subjects, 115 (28.6\%) were over the age of 14 years. All 115 participants consented to join the study; however, $1(0.88 \%)$ was lost to followup (Figure 2). This participant had no defining clinical or sociodemographic characteristics that stood apart from the rest of the cohort. Of the 114 patients over the age of 14 years remaining in the study, $55(48 \%)$ were seen during the dry season and $59(52 \%)$ during the wet season (Table 1). At enrolment, $80(72 \%)$ of patients reported coming to the clinic because of fever and of those, 37 (46.3\%) were febrile when tested by clinic staff. The remaining 34 participants $(29.8 \%)$ did not report fever as a main reason for seeking care but were febrile when tested by clinic staff. Patient outcomes for those under the age of 14 years are reported elsewhere7.

The majority of patients $(80 \%)$ reported resolution of symptoms at 14 -day follow-up $(n=90)$ with the rest $(n=24)$ reporting no change in symptoms. None of the patients in the study died or were referred for further care. Patients were primarily women $(84 \%)$, and the median age of participants was 30.0 years (interquartile range [IQR]: 21.0-40.8), with a slight difference in age between patients who improved (28.5 years) compared with patients who reported no symptom resolution (35 years) (UOR: 1.03; 95\% CI: 1.00-1.05). There were no differences between those who improved versus those who did not by season; roughly $50 \%$ of patients improved during each season, with the other half reporting no improvement during the follow-up period.

Only $16.7 \%$ of participants were new GAIA patients, with over $82 \%$ having already visited one of the clinics in the past year, though there was no association between these variables and patient outcomes. Patients were less likely to improve during the follow-up period if they reported commercial farming as their primary source of income compared with casual labour (UOR: 1.43; 95\% CI: 1.43-16.44). Only four participants lived less than an hour from a government clinic, and participants were significantly more likely to have not gotten better if they lived more than 1 hour from the nearest GAIA clinic.

Acute respiratory illness was the most frequent diagnosis given by the mobile clinic clinicians $(37.7 \%)$, followed by fever of unknown etiology $(21.9 \%)$ and general body pain $(16.7 \%)$ (Figure 3). The majority of patients $(86 \%)$ received one diagnosis and $13.2 \%$ received two diagnoses (Table 2 ). There was no significant difference between the number of 
diagnoses given to those who improved versus those who did not. Almost all patients received some type of medication during their clinic visit $(98.2 \%)$. Antibiotics were given to $38.6 \%$ of patients, with no significant difference between those who improved and those who did not. Virtually all patients received pain relief medication to either relieve pain or reduce fever $(96.5 \%)$, with no significant difference between those who improved and those who did not.

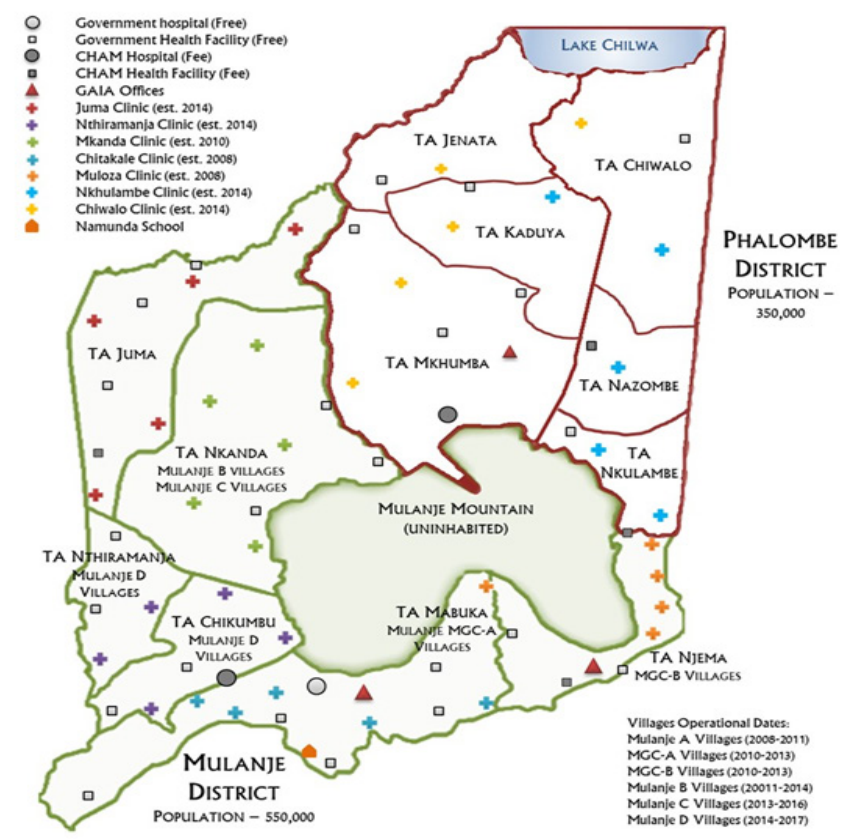

Figure 1: Map of mobile clinic sites in Mulanje and Phalombe districts

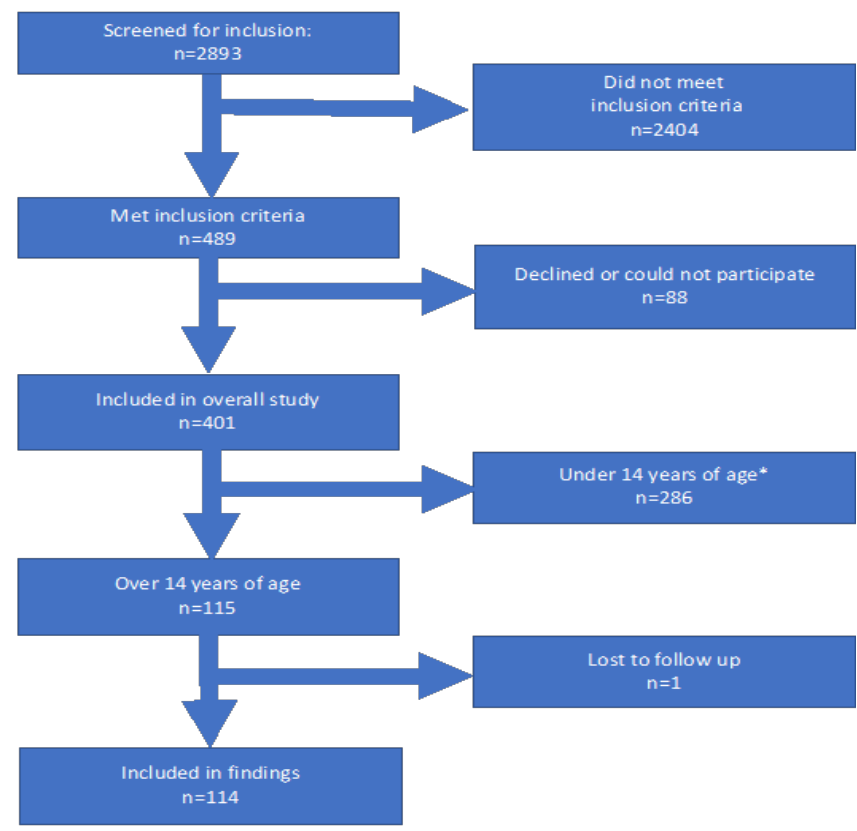

Figure 2: Flowchart of eligible participants presenting at a mobile clinic with a non-malarial fever in the wet or dry seaso

Those who did not report getting better were significantly more likely to self-report presenting to the clinic with non-specific ailment of 'looking or feeling poorly' (UOR: 12.71; 95\% CI: 1.54-264.16) and receive a clinical diagnosis of a general neurological issue (Figure 4). Of those who did not get better, only $54.2 \%$ believed their diagnosis compared with $82.5 \%$ of those who did get better (UOR: 0.14; 95\% CI: 0.04-0.45), and a quarter of those who did not get better also sought medication elsewhere during

\section{PARTICIPANTS WITH NO CHANGE OR} WORSENING
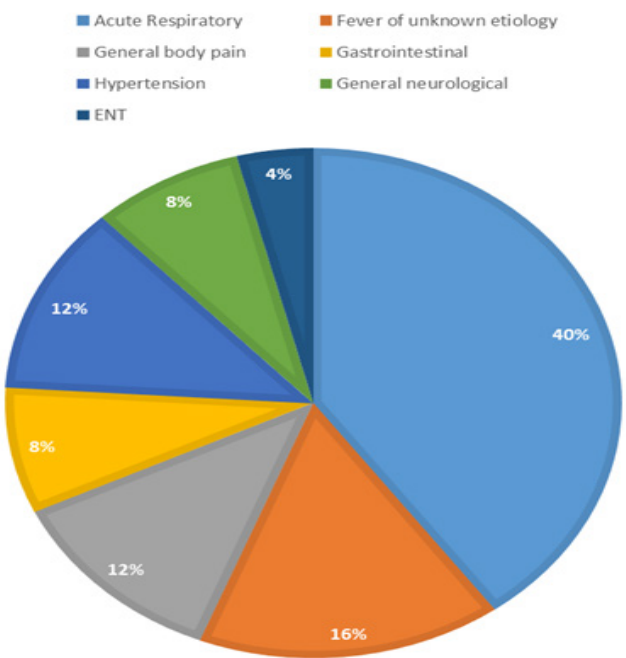

Figure 3: Clinician diagnoses for eligible participants presenting with non-malarial fevers based on their status at follow up

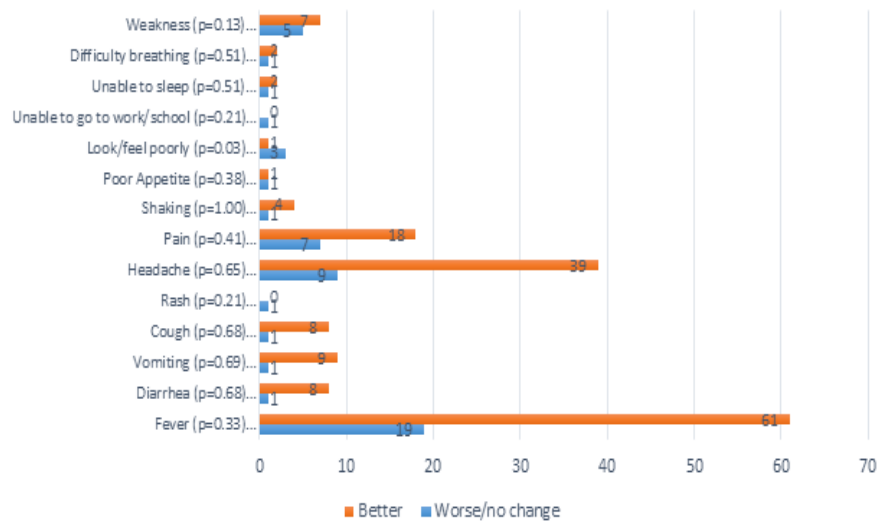

Figure 4: Presenting complaint by outcome status at 14 day follow up with associate $p$-value, odds ratio (OR) and $95 \%$ confidence interval

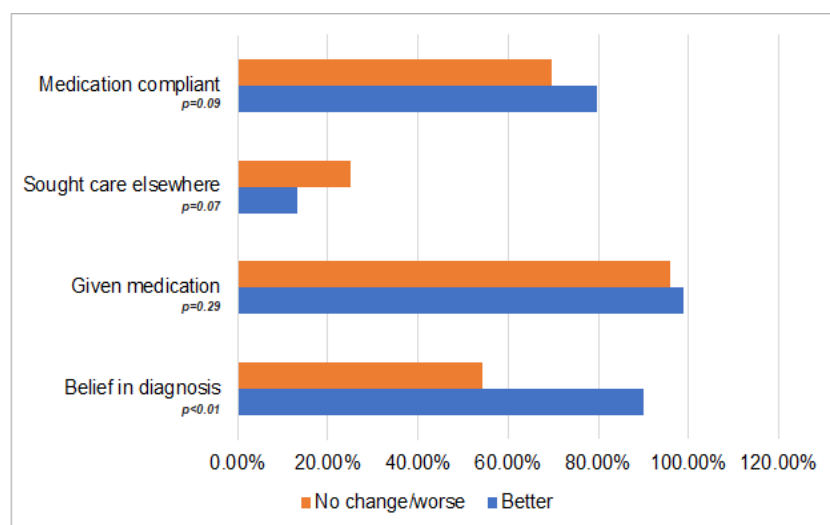

Figure 5. Bar plot of factors associated with medical treatment for participants based on their outcome at follow up

the 14-day follow-up period compared with $13.30 \%$ for those who got better, though this latter difference was not significant (Figure 5). There was no significant difference in the resolution of symptoms for those who sought care or medication elsewhere than the GAIA clinics. Though there was no significant difference in the overall amalgamated measurement of medication compliance (UOR: 0.52; 95\% CI: 0.19-1.52), within this measurement, those who did not get better were significantly more likely to have forgotten to take the medication over a weekend (UOR: 6.45; 95\% CI: 1.01-51.48). 
Table 1: Participant demographic characteristics overall and by symptom status at follow-up with associated(UOR), 95\%CI, and p-value

\begin{tabular}{|c|c|c|c|c|c|c|}
\hline & Total & Better & No change & \multirow[b]{2}{*}{ UOR } & \multirow[b]{2}{*}{$95 \% \mathrm{Cl}$} & \multirow[b]{2}{*}{ P-value } \\
\hline & $\mathrm{n}(\%)$ & $\mathrm{n}(\%)$ & $\mathrm{n}(\%)$ & & & \\
\hline TOTAL & $114(100 \%)$ & $90(78.9 \%)$ & $24(21.1 \%)$ & - & - & \\
\hline \multicolumn{7}{|l|}{ Season } \\
\hline Dry & $55(48.2 \%)$ & $44(48.9 \%)$ & $11(45.8 \%)$ & 0 & Ref & 0.82 \\
\hline Wet & $59(51.8 \%)$ & $46(51.1 \%)$ & $13(54.2 \%)$ & 1.13 & $0.46-2.83$ & \\
\hline \multicolumn{7}{|l|}{ Sex } \\
\hline Female & $96(84.2 \%)$ & $76(84.4 \%)$ & $20(83.3 \%)$ & 0 & Ref & 1 \\
\hline Male & $18(15.8 \%)$ & $14(15.6 \%)$ & $4(16.7 \%)$ & 1.09 & $0.28-3.42$ & \\
\hline \multicolumn{7}{|l|}{ Age } \\
\hline Median (IQR) & $30.0(21.0-40.75)$ & $28.5(21.0-38.0)$ & $35(26.5-56.0)$ & 1.03 & $1.00-1.05 \dagger$ & 0.055 \\
\hline \multicolumn{7}{|l|}{ Marital status } \\
\hline Married & $66(57.9 \%)$ & $52(57.8 \%)$ & $14(58.3 \%)$ & 0 & Ref & 1 \\
\hline Unmarried & $48(42.1 \%)$ & $38(42.2 \%)$ & $10(41.7 \%)$ & 0.98 & $0.38-2.42$ & \\
\hline \multicolumn{7}{|l|}{ Level of education } \\
\hline None & $30(26.3 \%)$ & $23(25.6 \%)$ & $7(29.2 \%)$ & 0 & Ref & 0.93 \\
\hline Primary school & $74(64.9 \%)$ & $59(65.6 \%)$ & $15(62.5 \%)$ & 0.84 & $0.31-2.43$ & \\
\hline Secondary school & $10(8.8 \%)$ & $8(8.9 \%)$ & $2(8.3 \%)$ & 0.82 & $0.11-4.32$ & \\
\hline \multicolumn{7}{|l|}{ Source of income } \\
\hline Casual labour & $40(35.1 \%)$ & $36(40.0 \%)$ & $4(16.7 \%)$ & 0 & Ref & 0.16 \\
\hline Commercial farming & $49(43.0 \%)$ & $33(36.7 \%)$ & $16(66.7 \%)$ & 4.36 & $1.43-16.44 \dagger$ & \\
\hline Selling food or produce & $6(5.3 \%)$ & $5(5.6 \%)$ & $1(4.2 \%)$ & 1.8 & $0.08-15.75$ & \\
\hline Supported by family & $3(2.6 \%)$ & $2(2.2 \%)$ & $1(4.2 \%)$ & 4.5 & $0.19-59.16$ & \\
\hline Small shop or business & $3(2.6 \%)$ & $3(3.3 \%)$ & $0(0.0 \%)$ & 0 & - & \\
\hline Tearoom/restaurant & $1(0.9 \%)$ & $1(1.1 \%)$ & $0(0.0 \%)$ & 0 & - & \\
\hline Other & $12(10.5 \%)$ & $10(11.1 \%)$ & $2(8.3 \%)$ & 1.8 & $0.23-10.72$ & \\
\hline \multicolumn{7}{|l|}{ First visit to GAIA clinic } \\
\hline Yes & $19(16.7 \%)$ & $16(17.8 \%)$ & $3(12.5 \%)$ & 0 & Ref & \\
\hline No & $95(83.3 \%)$ & $74(82.2 \%)$ & $21(87.5 \%)$ & 1.51 & $0.45-6.94$ & \\
\hline \multicolumn{7}{|l|}{ Distance from GAIA clinic } \\
\hline Less than 1 hour & $82(71.9 \%)$ & $70(77.8 \%)$ & $12(50.0 \%)$ & 0 & Ref & 0.15 \\
\hline $1-2$ hours & $26(22.8 \%)$ & $17(18.9 \%)$ & $9(37.5 \%)$ & 3.09 & $1.10-8.55 \dagger$ & \\
\hline $3-4$ hours & 0 & 0 & 0 & 0 & - & \\
\hline 5-6 hours & $1(0.9 \%)$ & $1(1.1 \%)$ & 0 & 8.75 & 1.32-71.99† & \\
\hline Don't know & $5(4.4 \%)$ & $2(2.2 \%)$ & $3(12.5 \%)$ & 0 & - & \\
\hline
\end{tabular}

$\mathrm{Cl}$, confidence interval; GAIA, Global AIDS Interfaith Alliance; IQR, interquartile range; Ref, reference value; UOR, unadjusted odds ratio.

Table 2. Number of recorded diagnoses and medications prescribed by GAIA and UOR, 95\% CI and P-values for measures of association

\begin{tabular}{|c|c|c|c|c|c|c|}
\hline \multirow{2}{*}{$\begin{array}{l}\text { Total number of recorded } \\
\text { diagnoses }\end{array}$} & Total & Better & No change & \multirow[b]{2}{*}{ UOR } & \multirow[b]{2}{*}{$95 \% \mathrm{Cl}$} & \multirow[b]{2}{*}{ P-value } \\
\hline & $\mathrm{n}(\%)$ & n (\%) & $\mathrm{n}(\%)$ & & & \\
\hline 0 & $1(0.9 \%)$ & $1(1.1 \%)$ & $0(0.0 \%)$ & 0 & - & 0.358 \\
\hline 1 & $98(86.0 \%)$ & $75(83.3 \%)$ & $23(95.8 \%)$ & 0 & Ref & \\
\hline 2 & $15(13.2 \%)$ & $14(15.6 \%)$ & $1(4.2 \%)$ & 0.23 & $0.01-1.26$ & \\
\hline \multicolumn{7}{|l|}{$\begin{array}{l}\text { Medication(s) prescribed } \\
\text { by GAIA }\end{array}$} \\
\hline \multicolumn{7}{|l|}{ Anti-epileptic } \\
\hline No & $111(97.4 \%)$ & $87(96.7 \%)$ & $24(100.0 \%)$ & 0 & Ref & 1 \\
\hline Yes & $3(2.6 \%)$ & $3(3.3 \%)$ & $0(0.0 \%)$ & - & - & \\
\hline \multicolumn{7}{|l|}{ Anti-helminth } \\
\hline No & $113(99.1 \%)$ & 89 (98.9\%) & $24(100.0 \%)$ & 0 & Ref & 1 \\
\hline Yes & $1(0.9 \%)$ & $1(1.1 \%)$ & $0(0.0 \%)$ & - & - & \\
\hline \multicolumn{7}{|l|}{ Anti-hypertensive } \\
\hline No & $109(95.6 \%)$ & $87(96.7 \%)$ & $22(91.7 \%)$ & 0 & Ref & 0.28 \\
\hline Yes & $5(4.4 \%)$ & $3(3.3 \%)$ & $2(8.3 \%)$ & 2.64 & $0.33-6.86$ & \\
\hline \multicolumn{7}{|l|}{ Antibiotic } \\
\hline No & $70(61.4 \%)$ & $54(60.0 \%)$ & $16(66.7 \%)$ & 0 & Ref & 0.64 \\
\hline Yes & $44(38.6 \%)$ & $36(40.0 \%)$ & $8(33.3 \%)$ & 0.75 & $0.28-1.89$ & \\
\hline \multicolumn{7}{|l|}{ Asthma drugs } \\
\hline No & $111(97.4 \%)$ & $87(96.7 \%)$ & $24(100.0 \%)$ & 0 & Ref & 1 \\
\hline Yes & $3(2.6 \%)$ & $3(3.3 \%)$ & $0(0.0 \%)$ & - & - & \\
\hline \multicolumn{7}{|l|}{ ORS } \\
\hline No & $108(94.7 \%)$ & $86(95.6 \%)$ & $22(91.7 \%)$ & 0 & Ref & 0.6 \\
\hline Yes & $6(5.3 \%)$ & $4(4.4 \%)$ & $2(8.3 \%)$ & 1.95 & $0.26-10.70$ & \\
\hline \multicolumn{7}{|l|}{ Pain reliever } \\
\hline No & $4(3.5 \%)$ & $2(2.2 \%)$ & $2(8.3 \%)$ & 0 & Ref & 0.19 \\
\hline Yes & $110(96.5 \%)$ & $88(97.8 \%)$ & $22(91.7 \%)$ & 0.25 & $0.03-2.18$ & \\
\hline \multicolumn{7}{|l|}{ Supplement } \\
\hline No & $108(94.7 \%)$ & $86(95.6 \%)$ & $22(91.7 \%)$ & 0 & Ref & 0.6 \\
\hline Yes & $6(5.3 \%)$ & $4(4.4 \%)$ & $2(8.3 \%)$ & 1.95 & $0.26-10.70$ & \\
\hline \multicolumn{7}{|l|}{$\begin{array}{l}\text { Total medications given } \\
\text { by GAIA }\end{array}$} \\
\hline 1 & $20(17.5 \%)$ & $15(16.7 \%)$ & $5(20.8 \%)$ & 0 & Ref & 0.32 \\
\hline 2 & $74(64.9 \%)$ & $57(63.3 \%)$ & $17(70.8 \%)$ & 0.89 & $0.30-3.07$ & \\
\hline 3 & $17(14.9 \%)$ & $16(17.8 \%)$ & $1(4.2 \%)$ & 0.19 & $0.01-1.34$ & \\
\hline 4 & $3(2.6 \%)$ & $2(2.2 \%)$ & $1(4.2 \%)$ & 1.5 & $0.06-19.35$ & \\
\hline \multicolumn{7}{|l|}{$\begin{array}{l}\text { Did the patient seek } \\
\text { care elsewhere after } \\
\text { GAIA }\end{array}$} \\
\hline No & $71(62.2 \%)$ & $59(66.3 \%)$ & $12(50.0 \%)$ & 0 & Ref & 0.22 \\
\hline Yes & $42(36.8 \%)$ & $30(33.7 \%)$ & $12(50.0 \%)$ & 1.97 & $0.79-4.90$ & \\
\hline
\end{tabular}

$\mathrm{Cl}$, confidence interval; GAIA, Global AIDS Interfaith Alliance; ORS, oral rehydration salts; Ref, reference value; UOR, unadjusted odds ratio. 


\section{Discussion}

Similar to findings from the paediatric subgroup in this parent study ${ }^{7}$, outcomes were excellent among adults in this setting, with $80 \%$ reporting resolution of symptoms at 14 days. However, occupation, distance from a mobile clinic, age and non-specific presenting complaint were associated with no change or worsening of symptoms among the participants in this study. The majority of participants who reported commercial farming as an occupation had symptoms that were unresolved or worsening during the 14-day follow-up period. In low- and middle-income countries, occupational hazards, including exposure to airborne particulates, are believed to contribute substantially to pulmonary disease? This potential exposure among commercial farmers in the study may partially explain the delayed improvement or no change in condition reported especially in light of the fact that acute respiratory illness was the most frequent diagnosis.

Outcomes were not influenced by seasonality, but patients who lived far from the mobile clinics were less likely to improve, similar to other findings in the same area as well as results from the paediatric subgroup in this study $y^{7,10}$. Although information on duration of symptoms prior to visiting the mobile clinics was not available, it is possible that those who live closer to the mobile clinic sites present earlier in an illness trajectory and, therefore, outcomes are better. Additionally, patients in this study who were older were less likely to improve, and although non-communicable diseases are thought to be increasingly responsible for morbidity in older adults ${ }^{11}$, infectious disease remains a primary driver of illness in rural Malawi.

Similar to other non-malarial fever studies, this study found that most non-malarial fevers were assigned a diagnosis of respiratory illness and the majority of patients were given analgesics, followed by antibiotics ${ }^{12,13}$. None of the adult patients in this study were given anti-malarials, unlike similar settings where providers may prescribe anti-malarials despite a negative $\mathrm{mRDT}^{14}$.

The significant association between distance to care and patient outcomes highlights the critical role that mobile clinics such as those of GAIA can play in the developing world. In a setting with no public transportation and where almost all rural inhabitants walk to clinics, only $3.5 \%$ of participants were less than an hour from a government clinic, with over 50\% more than 3 hours away. In contrast, $71.9 \%$ of participants were less than an hour from a GAIA clinic. Interestingly, there was no significant association among participants between the number of prior visits to GAIA and their distance to the mobile clinics, potentially due to the fact that all but one participant lived within less than 2 hours of a clinic, making care accessible when needed. In underdeveloped regions where resources are scarce, patients may be unable to seek timely care due to financial or temporal reasons, allowing diseases to progress to a less easily treatable stage.

Despite being mobile clinics without the benefit of testing facilities associated with larger stationary facilities, the vast majority of patients seen at GAIA experienced symptom resolution following their visit. It is not surprising that those who did not get better were less likely to believe their diagnoses due to the perpetuation of their concerns. However, their ongoing complaints do not appear to be the result of incorrect diagnoses or treatment as they were no more likely to get better when seeking care elsewhere than the mobile clinics. Instead, their lack of resolution may be associated with their less specific presenting complaint and/or generalised diagnosis. While these patients, due to their unresolved poor health, need to be considered when ramping up the diagnostic and treatment capabilities of mobile clinic facilities, they suggest that current testing and treatment algorithms used are successful and appropriate for the majority of patients served.

The study has several limitations worth noting. First, the follow-up period was 14 days, which may have prevented the researchers from capturing longer-term issues related to the underlying illnesses. In the future, an extended follow-up period may allow for a more nuanced understanding of nonmalarial febrile illness, particularly around health-seeking behaviours outside the mobile clinics. Further, the majority of the patients in this study were women, limiting the ability to generalise to male populations. It is possible that men may seek care less often than women due to work responsibilities, because the mobile clinics visit sites during daytime working hours. It is interesting to note that this study did find commercial farming to be a risk for unresolved illness; therefore, future studies may seek to specifically enrol men with this occupation.

\section{Conclusions}

Despite many concerted efforts, the provision of equitable healthcare to rural populations in the developing world continues to face many structural, temporal and financial barriers. These are in addition to concerns that the care provided is of the highest quality. Rapid point-of-care testing kits and immediate provision of treatment for diseases such as HIV and malaria have been critical to addressing many of these issues. However, challenges remain when a patient's presenting complaint is less well defined. This study sought to assess the characteristics, diagnoses, treatment and outcomes in patients with mRDT-negative fevers presenting at mobile clinics in rural Malawi through a prospective cohort. The findings demonstrated that the clinics were by-and-large successful at addressing a wide variety of symptoms associated with a non-malarial fever leading to an improvement or complete resolution of symptoms. Although infectious disease remains an area where simple diagnostics facilitate sound treatment decisions, as the average lifespan increases in this setting, providers may need additional training in chronic diseases.

\section{Availability of data and materials}

The datasets analysed during the current study are available from the corresponding author on reasonable request.

\section{Competing interests}

The authors declare that they have no competing interests.

\section{References}

1. Burchett HED, Leurent B, Baiden F, Baltzell K, Björkman A, Bruxvoort K, et al. Improving prescribing practices with rapid diagnostic tests (RDTs): synthesis of 10 studies to explore reasons for variation in malaria RDT uptake and adherence. BMJ Open. 2017;7(3):e012973. doi: 10.1136.bmjopen-2016-012973.

2. Zurovac D, Rowe AK. Quality of treatment for febrile illness among children at outpatient facilities in sub-Saharan Africa. Ann Trop Med Parasitol. 2006;100(4):283-296.

3. WHO | T3: Test. Treat. Track initiative [Internet]. $2012 \mathrm{WHO}$ [cited 2017 Jul 3]. Available from: http://www.who.int/malaria/areas/test_ treat_track/en/. 
4. Chappuis F, Alirol E, d'Acremont V, Bottieau E, Yansouni CP. Rapid diagnostic tests for non-malarial febrile illness in the tropics. Clin Microbiol Infect. 2013;19(5):422-431. doi: 10.1111/1469-0691.12154.

5. WHO informal consultation on fever management in peripheral health care settings: A global review of evidence and practice [Internet]. World Health Organization; 2013 [cited 2017 June 28]. Available from: http://apps.who.int/iris/bitstream/10665/95116/1/9789241506489_eng. pdf.

6. US Centers for Disease Control and Prevention. National action plan for combating antibiotic-resistant bacteria [Internet]. US Centers for Disease Control and Prevention; 2015 [cited 2017 June 15]. Available from: www.cdc.gov/drugresistance/pdf/national_action_plan_for_ combating_antibiotic-resistant_bacteria.pdf.

7. Kortz TB, Blair A, Scarr E, Mguntha AM, Bandawe G, Schell E, et al. Characterizing pediatric non-malarial fever and identifying the at-risk child in rural Malawi. Glob Pediatr Health. 2018;5. doi: 10.1177/2333794X17750415.

8. Tadesse E, Workalemahu B, Shimelis T. Diagnostic performance evaluation of the SD Bioline Malaria Antigen Ag Pf/Pan test (05fk60) in a malaria endemic area of southern Ethiopia. Rev Inst Med Trop Sao Paulo. 2016 Sep 22;58:59.

9. Global Health Risks [Internet]. World Health Organization; 2018 [cited 2017 May 20]. Available from: www.who.int/healthinfo/global_ burden_disease/global_health_risks/en/.
10. Ewing VL, Lalloo DG, Phiri KS, Roca-Feltrer A, Mangham LJ, SanJoaquin MA. Seasonal and geographic differences in treatmentseeking and household cost of febrile illness among children in Malawi. Malar J. 2011;10:32. doi: 10.1186/1475-2875-10-32.

11. GBD 2015 DALYs and HALE Collaborators. Global, regional, and national disability-adjusted life-years (DALYs) for 315 diseases and injuries and healthy life expectancy (HALE), 1990-2015: a systematic analysis for the Global Burden of Disease Study 2015. Lancet. 2016;388(10053):1603-1658. doi: 10.1016/S0140-6736.

12. Saweri OPM, Hetzel MW, Mueller I, Siba PM, Pulford J. The treatment of non-malarial febrile illness in Papua New Guinea: findings from cross sectional and longitudinal studies of health worker practice. BMC Health Serv Res. 2017 05;17(1):10.

13. Hildenwall H, Amos B, Mtove G, Muro F, Cederlund K, Reyburn H. Causes of non-malarial febrile illness in outpatients in Tanzania. Trop Med Int Health TM IH. 2016;21(1):149-156. doi: 10.1111/tmi.12635.

14. Altaras R, Nuwa A, Agaba B, Streat E, Tibenderana JK, Strachan CE. Why do health workers give anti-malarials to patients with negative rapid test results? A qualitative study at rural health facilities in western Uganda. Malar J. 2016;15:23. doi: 10.1186/s12936-015-1020-9. 